\title{
BMJ Open Psychological interventions for positive symptoms in schizophrenia: protocol for a network meta-analysis of randomised controlled trials
}

Irene Bighelli, ${ }^{1}$ Georgia Salanti, ${ }^{2}$ Cornelia Reitmeir, ${ }^{1}$ Sofia Wallis, ${ }^{1}$ Corrado Barbui, ${ }^{3}$ Toshi A Furukawa, ${ }^{4}$ Stefan Leucht ${ }^{1}$

To cite: Bighelli I, Salanti G, Reitmeir C, et al. Psychological interventions for positive symptoms in schizophrenia: protocol for a network metaanalysis of randomised controlled trials. BMJ Open 2018;8:e019280. doi:10.1136/ bmjopen-2017-019280

- Prepublication history and additional material for this paper are available online. To view these files, please visit the journal online (http://dx.doi. org/10.1136/bmjopen-2017019280).

Received 22 August 2017 Revised 28 December 2017 Accepted 23 January 2018

\section{Check for updates}

${ }^{1}$ Department of Psychiatry and Psychotherapy, Klinikum rechts der Isar, Technischen Universitat Munchen, Munchen, Germany ${ }^{2}$ Institute of Social and Preventive Medicine (ISPM), University of Bern, Bern,

Switzerland

${ }^{3}$ WHO Collaborating Centre for Research and Training in Mental Health and Service Evaluation, Department of Neuroscience, Biomedicine and Movement Sciences, Section of Psychiatry, University of Verona, Verona,

Italy

${ }^{4}$ Department of Health

Promotion and Human Behavior, Kyoto Graduate School of

Medicine/School of Public

Health, Kyoto University, Kyoto, Japan

Correspondence to

Dr Irene Bighelli;

irene.bighelli@tum.de

\section{ABSTRACT}

Introduction There is rising awareness that we need multidisciplinary approaches integrating psychological treatments for schizophrenia, but a comprehensive evidence based on their relative efficacy is lacking. We will conduct a network meta-analysis (NMA), integrating direct and indirect comparisons from randomised controlled trials (RCTs) to rank psychological treatments for schizophrenia according to their efficacy, acceptability and tolerability. Methods and analysis We will include all RCTs comparing a psychological treatment aimed at positive symptoms of schizophrenia with another psychological intervention or with a no treatment condition (waitinglist and treatment as usual). We will include studies on adult patients with schizophrenia, excluding specific subpopulations (eg, first-episode patients or patients with psychiatric comorbidities). Primary outcome will be the change in positive symptoms on a published rating scale. Secondary outcomes will be acceptability (dropout), change in overall and negative symptoms of schizophrenia, response, relapse, adherence, depression, quality of life, functioning and adverse events. Published and unpublished studies will be sought through database searches, trial registries and websites. Study selection and data extraction will be conducted by at least two independent reviewers. We will conduct random-effects NMA to synthesise all evidences for each outcome and obtain a comprehensive ranking of all treatments. NMA will be conducted in Stata and $R$ within a frequentist framework. The risk of bias in studies will be evaluated using the Cochrane Risk of Bias tool and the credibility of the evidence will be evaluated using an adaptation of the Grading of Recommendations Assessment, Development and Evaluation framework to NMA, recommended by the Cochrane guidance. Subgroup and sensitivity analyses will be conducted to assess the robustness of the findings. Ethics and dissemination No ethical issues are foreseen Results from this study will be published in peer-reviewed journals and presented at relevant conferences. PROSPERO registration number CRD42017067795.

\section{INTRODUCTION}

Schizophrenia is a debilitating and often lifelong disorder that ranks among the top 20 causes of disability according to the World
Strengths and limitations of this study

- We will conduct a random-effects network metaanalysis (NMA) to synthesise all available published or unpublished randomised controlled trials for each prespecified outcome and obtain a comprehensive ranking of all treatments.

- This will be the first NMA on psychological treatments for schizophrenia; the findings from this study have the potential to inform and influence clinical decision-making and guideline development.

- The risk of heterogeneity and inconsistency is high, given the different psychological interventions that will be included: however, we try to control variability by carefully framing the inclusion criteria about population and interventions, and we will evaluate consistency employing local as well as global methods.

- The limitations of primary studies will be addressed with the Cochrane risk of bias tool, and the quality of evidence for network estimates will be assessed with an appropriate adaptation of the Grading of Recommendations Assessment, Development and Evaluation framework; these approaches are considered the gold standard for critical appraisal of evidence quality.

Health Report. ${ }^{1}$ Although pharmacological interventions have been the mainstay of treatment for schizophrenia, antipsychotics have a number of limitations (limited response, high incidence of disabling side effects and poor adherence to treatment $)^{2}$ and are problematic in many situations (such as medical comorbidities, tolerability problems and pregnancy). Besides, there has been growing recognition of the importance of psychological processes in psychosis, both as contributors to onset and persistence, and in terms of the negative psychological impact of a diagnosis of schizophrenia on the individual's well-being, psychosocial functioning and life opportunities. Psychological interventions 
for psychosis and schizophrenia have been developed to address these aspects, and in accordance with guidelines from the National Institute for Health and Care Excellence in the $\mathrm{UK}^{3}$ and the Schizophrenia Patient Outcomes Research Team in the USA, ${ }^{4}$ psychological treatments are widely regarded as a necessary intervention for schizophrenia.

A broad range of interventions that can be defined as 'psychological' have been studied in the treatment of schizophrenia. These interventions can be provided at different stages of the illness and address different aspects, like social and cognitive functioning, adherence to medication and symptoms of schizophrenia. Table 1 presents the panorama of existing systematic reviews of randomised controlled trials (RCTs) that have been conducted on the topic. These reviews have mainly included studies comparing the intervention under examination with so called no treatment conditions (waiting-list and treatment as usual (TAU)). ${ }^{56}$ Other reviews included also active comparisons with other psychological treatments. ${ }^{7-9}$ An attempt to provide information on active comparisons was made by Turner and colleagues, who performed separate meta-analysis when there were at least five eligible RCTs comparing one intervention to another psychological intervention. ${ }^{10}$ However, all the available reviews applied pairwise meta-analysis as a method, being able to pool results only when a comparison of two treatments was considered in existing studies. The comparative efficacy and tolerability of the existing interventions have not been checked yet; as a result, it is still currently unclear which are the most efficacious, the most acceptable and the best tolerable psychological treatments for schizophrenia.

To overcome this gap in the current knowledge, a NMA would be necessary to consider both direct and indirect comparisons, and produce hierarchies of the effects of the various psychological treatments in the various efficacy and tolerability outcomes. Such hierarchies are essential for guidelines, which should ideally be able to indicate which treatment is likely to be the best, the second best and so on for a given outcome. Only the method of NMA can provide such hierarchies by combining all the randomised evidence. Our aim is to produce such a NMA of all psychological interventions for schizophrenia in multiple outcomes. We focus here on the interventions primarily aimed at treating positive symptoms in the acute phase of the illness.

\section{Objectives}

To estimate relative treatment effects and obtain a hierarchy for the psychological treatments in patients with schizophrenia, in terms of:

1. efficacy on positive symptoms.

2. acceptability.

3. other efficacy measures, such as overall symptoms, negative symptoms, response, relapse, adherence, depression, quality of life and functioning.

4. tolerability.

\section{METHODS AND ANALYSIS}

\section{Criteria for considering studies for this review}

Methods for this systematic review have been developed according to the Preferred Reporting Items for Systematic review and Meta-Analysis Protocols (PRISMA-P) checklist, and the PRISMA extension statement for reporting of systematic reviews incorporating NMA of healthcare interventions. ${ }^{11} 12$ This systematic review and NMA is registered in the PROSPERO database; the record in PROSPERO will be updated with any amendment made to the protocol.

\section{Types of studies}

We will include all RCTs in which participants with schizophrenia received a psychological intervention as defined below (see Types of interventions section). Studies whose sequence generation was at high risk of bias (eg, randomisation by the date of birth or day of the week) will be excluded. We will accept open and blinded RCTs; this choice is particularly relevant in trials on psychological interventions, in which in best case only the assessor of outcome can be blind, but not the therapist. Open RCTs will be excluded in a sensitivity analysis. We will include both trials in which psychological interventions were compared with a control condition and trials in which they were compared with another intervention. There will be no language restriction in order to avoid the problem of 'language bias'. ${ }^{13}$ In case we retrieve references in languages in which we are not fluent, study authors will be contacted to check inclusion criteria and eventually ask for study data. As an exception, we will not search Chinese databases, since serious concerns have been raised on the trustworthiness of Chinese trials found in these databases. ${ }^{1415}$ Chinese studies found in Western databases will be considered for inclusion. In the case of cross-over studies, we will use only the first cross-over phase in order to avoid the problem of carry-over effects which are very likely in schizophrenia and with psychological treatments. We will exclude cluster RCTs.

\section{Types of participants}

Our aim is to collect information on the efficacy of psychological treatments on patients with positive symptoms. In order to select this population, we operationalised the inclusion criteria as follows. We will include adults, however defined by study authors, with a diagnosis of schizophrenia or related disorders (such as schizophreniform or schizoaffective disorders); there is no clear evidence that the latter schizophrenia-like psychoses are caused by fundamentally different disease processes or require different treatment approaches. ${ }^{16}$ We will include trials irrespective of the diagnostic criteria used. Here we will follow the strategy of the Cochrane Schizophrenia Group $^{17}$ to include not only studies that used specific diagnostic criteria such as International Classification of Diseases, 10th revision or Diagnostic and Statistical Manual of Mental Disorders, 5th edition, because these criteria are not meticulously used in clinical routine 
Table 1 Existing reviews about psychological treatments for schizophrenia

\begin{tabular}{|c|c|c|c|}
\hline Intervention & Existing reviews & $\mathbf{R C T}^{*}$ & Comparator \\
\hline $\begin{array}{l}\text { Acceptance and commitment } \\
\text { therapy }\end{array}$ & Ongoing Cochrane review ${ }^{42}$ & - & $\begin{array}{l}\text { TAU, pharmacological intervention and another } \\
\text { psychosocial intervention }\end{array}$ \\
\hline Adherence interventions & Gray et $a l^{43}$ & 6 & TAU and didactic health education \\
\hline $\begin{array}{l}\text { Active comparisons (befriending, } \\
\text { CBT, cognitive remediation, } \\
\text { psychoeducation, social skills } \\
\text { training, supportive counselling) }\end{array}$ & Turner et $a l^{10}$ & 48 & $\begin{array}{l}\text { Befriending, CBT, cognitive remediation, psychoeducation, } \\
\text { social skills training, supportive counselling, family } \\
\text { intervention, art therapy, body psychotherapy, } \\
\text { occupational therapy and problem-solving therapy }\end{array}$ \\
\hline Assertive community treatment & Marshall and Lockwood ${ }^{44}$ & $20 \dagger$ & TAU, hospital-based rehabilitation and case management \\
\hline Befriending & - & & \\
\hline Bibliotherapy & - & & \\
\hline $\begin{array}{l}\text { Body-oriented psychological } \\
\text { therapy }\end{array}$ & - & & \\
\hline
\end{tabular}

\begin{tabular}{|c|c|c|c|}
\hline Case management & Dieterich et a $\left.\right|^{45}$ & $40 \dagger$ & $\begin{array}{l}\text { Assertive community treatment, assertive outreach model, } \\
\text { case management model and standard community care }\end{array}$ \\
\hline CBT & $\begin{array}{l}\text { Zimmermann et al (positive } \\
\text { symptoms) }^{9}\end{array}$ & 15 & Waiting-list, TAU or another therapeutic treatment \\
\hline
\end{tabular}

Jones et $\mathrm{al}^{7}$

20

Jauhar et $a l^{46}$

52

Van der Gaag et al (individually tailored) $^{47}$

Hazell et al (low intensity) ${ }^{48} \quad 8$

Kennedy and Xyrichis 2 (auditory hallucinations) $^{49}$

\begin{tabular}{|c|c|c|c|}
\hline Cognitive remediation & Cella et $a^{50}$ & 45 & $\begin{array}{l}\text { TAU, active control (eg, computer games) another active } \\
\text { treatment (eg, CBT) }\end{array}$ \\
\hline Dance therapy & Ren and $\mathrm{Xia}^{51}$ & 1 & Standard care plus supportive counselling \\
\hline \multirow[t]{2}{*}{ Family interventions } & Pitschel-Walz et al ${ }^{52}$ & 25 & TAU, patient intervention, other family interventions \\
\hline & Pharoah et $a l^{53}$ & 25 & $\begin{array}{l}\text { TAU, discussion groups, psychoeducation, supportive } \\
\text { psychotherapy, psychosocial support }\end{array}$ \\
\hline $\begin{array}{l}\text { Group psychotherapeutic } \\
\text { treatments }\end{array}$ & Orfanos et $a l^{54}$ & 34 & $\begin{array}{l}\text { TAU and other groups (active discussion group, support } \\
\text { group, counselling group, occupational therapy group or } \\
\text { problem-solving discussion group) }\end{array}$ \\
\hline IPT & Roder et $a^{55}$ & 16 & $\begin{array}{l}\text { TAU, placebo-attention condition and other active } \\
\text { treatments }\end{array}$ \\
\hline $\begin{array}{l}\text { Psychological and psychosocial } \\
\text { interventions for negative } \\
\text { symptoms in psychosis }\end{array}$ & Lutgens et $a l^{8}$ & 95 & $\begin{array}{l}\text { TAU and active comparator (including psychoeducation, } \\
\text { supportive therapy and cognitive remediation) }\end{array}$ \\
\hline Music therapy & Geretsegger et a/ ${ }^{58}$ & 18 & $\begin{array}{l}\text { Placebo defined as an alternative therapy designed to } \\
\text { control for effects of the therapist's attention; TAU or no } \\
\text { treatment }\end{array}$ \\
\hline
\end{tabular}

Continued 
Table 1 Continued

\begin{tabular}{|c|c|c|c|}
\hline Intervention & Existing reviews & $\mathrm{RCT}^{*}$ & Comparator \\
\hline Psychodynamic therapy & Malmberg and Fenton 59 & 4 & $\begin{array}{l}\text { Reality adaptive, supportive psychotherapy, hospital } \\
\text { comparison, ataraxic drugs, electro convulsive therapy, } \\
\text { Milieu therapy and individual versus group }\end{array}$ \\
\hline Psychoeducation & Pekkala and Merinder ${ }^{60}$ & 10 & $\begin{array}{l}\text { TAU, supportive psychotherapy, behavioural intervention } \\
\text { and leisure-time group }\end{array}$ \\
\hline Social skills training & Almerie et $a l^{61}$ & 13 & $\begin{array}{l}\text { TAU, structured activities, discussion group, interaction } \\
\text { group, and no treatment control }\end{array}$ \\
\hline Systemic therapy & Pinquart et $a l^{5}$ & 7 & No treatment \\
\hline
\end{tabular}

${ }^{*}$ Number of RCTs on patients with schizophrenia .

†RCTs about patients with severe mental illness including schizophrenia.

CBT, cognitive-behavioural therapy; IPT, integrated psychological therapy; RCT, randomised controlled trial; TAU, treatment as usual.

either. This decision should increase generalisability and representativeness.

Studies including participants with other diagnoses part of the psychosis spectrum will be included only if participants with a diagnosis of schizophrenia, schizophreniform or schizoaffective disorders were $>80 \%$ of the participants considered. We will include studies recruiting patients with positive symptoms, either delusions, hallucinations or both, or in the phase of acute exacerbation of positive symptoms, however defined by inclusion criteria of the trial.

We will exclude studies focused on specific subpopulations of patients, such as (A) studies recruiting patients in which negative symptoms are predominant, according to authors' definition, (B) studies on patients with comorbid psychiatric disorders including substance abuse, (C) studies recruiting patients with concomitant medical illnesses, (D) trials enrolling stable patients (relapse prevention studies), (E) studies on first-episode patients and $(\mathrm{F})$ trials on patients who show prodromal signs of psychosis (also defined as 'at risk for psychosis'). Among other reasons, we exclude first-episode patients because they were found to have significantly higher response rates to treatments compared with chronic patients. ${ }^{18} 19$

\section{Types of interventions}

Any psychological intervention that occurs through interaction between therapist and patient, either face-to-face individually or in group, with the primary aim to reduce positive symptoms. Interventions with an explicit primary aim different from positive symptoms (eg, functioning, cognition, adherence to medication and knowledge of the illness) will be excluded. The identified treatments will be classified after identification of eligible studies. Psychological treatments will be compared with each other and to any non-pharmacological control condition considered in the included studies. Comparators will include the so called 'treatment as usual', waiting-list and inactive treatments. The effect of 'non-active' comparators will be analysed in a sensitivity analysis. ${ }^{20}$ Patients also receiving treatment as usual, including pharmacological interventions, will be included. If psychological treatments that we do not include among the interventions (eg, psychoeducation and supportive therapy) are used as control condition in the studies, they will be included as nodes in order to strengthen the network, but will not be part of our decision set.

\section{Outcome measures}

Outcomes will be measured at study endpoint, as defined in each study.

\section{Primary outcome}

Change in positive symptoms of schizophrenia, examined accordingly to the respective subscale of the Positive and Negative Syndrome Scale (PANSS), the Brief Psychiatric Rating Scale (BPRS) or the Scales for Assessment of Positive Symptoms or any other published scale.

As not all studies will have used the same scale, we will extract data according to the following hierarchy: mean change of the PANSS positive symptoms subscale from baseline to endpoint, if not available mean change of the BPRS positive symptoms subscale or if again not available the mean values at endpoint of the PANSS/ BPRS positive symptoms subscale. The results of other rating scales will only be used if the instrument has been published in a peer-reviewed journal, because it has been shown that non-validated schizophrenia scales exaggerate differences. ${ }^{21}$

\section{Secondary outcomes}

Given the focus on treatments for positive symptoms, the results of this review will be informative for the treatment of positive symptoms. They will also describe how these interventions can have an effect on a number of other outcomes. With this aim, the following secondary outcomes will be assessed: 
1. Acceptability, defined as the percentage of patients leaving the study early (dropout) for any reason. Allcause discontinuation due to any reason combines efficacy, tolerability and other factors, and can therefore be considered as a measure of 'acceptability of treatment ${ }^{17}$ or of overall 'effectiveness'.

2. Change in overall symptoms, measured by rating scales such as the PANSS or the BPRS, or any other published scale (eg, the Manchester Scale) for the assessment of overall schizophrenic symptomatology. The results of other rating scales will only be used if the instrument has been published in a peer-reviewed journal.

3. Change in negative symptoms, measured by the respective subscale of the PANSS, or the 'Scales for Assessment of Negative Symptoms' or any other published scale.

4. Response, measured by the percentage of responders defined by reduction on the PANSS, BPRS or Clinical Global Impression (CGI) scores, accepting the criteria used by study authors.

5. Percentage of patients with relapse, by definitions operationalised by rating scales, and, if not available, number of rehospitalisations due to psychopathology. We will not include data from studies that used non-operationalised relapse criteria (eg, clinical judgement).

6. Adherence, measured by any published rating scale (eg, 'Adherence Therapy Patients Satisfaction Questionnaire' and 'Adherence Rating Scale').

7. Depression, measured by the Calgary Depression Scale for Schizophrenia, the Hamilton Depression Rating Scale, the Montgomery Asberg Depression Scale or other published symptom scales.

8. Quality of life, measured by any published rating scale (eg, 'Heinrichs quality of life scale', Quality of Life Scale.

9. Functioning, measured by rating scales such as the Global Assessment of Functioning or the Psychosocial Performance Scale, or any other published rating scale.

10. Tolerability, measured as the percentage of patients experiencing adverse events. Adverse events associated with psychological treatments are not covered as comprehensively as in trials on pharmacological treatments. ${ }^{22}$ However, there is a raising awareness of the importance of considering possible harms associated with psychological interventions. ${ }^{23}$ Therefore we will collect any available information in clinical studies about this outcome, using a classification proposed by Linden and colleagues ${ }^{23}$ : (A) emergence of new symptoms; (B) deterioration of existing symptoms; (C) lack of improvement or deterioration of illness; (D) prolongation of treatment; (E) patient's non-compliance; (F) strains in the patient-therapist relationship; $(\mathrm{G})$ very good patient-therapist relationship, therapy dependency; $(\mathrm{H})$ strains or changes in family relations; (I) strains or changes in work relations; ( $\mathrm{J}$ ) any change in the life circumstances of the patient; (K) stigmatisation. Suicide attempts and any other possible adverse event related to psychological treatment will also be considered.

11. Mortality. Psychosocial treatments may actually reduce or, by contrast, increase overall mortality, in particular connected to suicidality. To test this, we will examine this outcome in terms of (A) death for any reason, (B) death due to natural causes and (C) due to suicide.

\section{Search strategy}

\section{Electronic searches}

The following sources will be searched without restrictions for language or publication period: Embase, MEDLINE, PsycINFO and PubMed. The search terms that will be used for PubMed are provided as online supplementary material. We will also search the following international databases:

1. WHO International Clinical Trials Registry Platform,

2. BIOSIS,

3. Cochrane Collaboration Controlled Trials Register,

4. ClinicalTrials.gov.

\section{Reference lists and other sources}

References of all selected studies will be inspected for other published reports and citations of unpublished studies. We will also inspect previous reviews conducted on psychological treatments for schizophrenia to check if some studies meet our inclusion criteria as well. In addition, we will contact the first author of each included study published in the last 30 years for missing information about their studies.

\section{Identification and selection of studies}

Studies identified through electronic and manual searches will be listed with citation, titles and abstracts, in Citavi; duplicates will be excluded. The eligibility for inclusion process will be conducted in two separate stages:

1. Two authors will independently inspect title and abstracts identified in the literature searches and exclude those not pertinent. Disagreement will be resolved by discussion and where doubt still remains, we will acquire the full article for further inspection and the article will proceed to the next stage.

2. Once the full articles are obtained, two reviewers will independently assess them for eligibility. Disagreements will be resolved by discussion and, if needed, a third senior author will be involved. When required, further information will be obtained from study authors.

\section{Data extraction}

Two authors will independently extract data from all selected trials. When disagreement arises we will resolve it by discussion and, if needed, involving a third senior author. Where this is not sufficient we will contact the study authors.

The following data will be collected from each included study: 
- Study citation, year(s) of study, registration number to trials registries, year of publication, location, setting, number of centres, sample size, diagnostic criteria and funding/sponsor (industry or academic).

- Methodology, including study design (type of RCT), number of arms and risk of bias (see Risk of bias assessment section).

- Characteristics of study participants, including gender, age, details on diagnosis, number randomised to each arm, sociodemographic characteristics, whether psychological treatments naive at baseline or with previous experience with the experimental intervention).

- Characteristics of intervention, including number and frequency of sessions, therapy setting, expertise of therapist and researcher allegiance at study arm level.

- Outcome measures, including information on whether an intention-to-treat approach has been used and how it was defined.

The two reviewers will independently input data into an Access database, especially created for this study. The software will automatically detect any inconsistencies and they will be resolved by discussion.

\section{Measurement of treatment effect \\ Relative treatment effects}

- Continuous outcomes: for continuous outcomes we will use the standardised mean difference (SMD), because we expect that the studies use different rating scales of overall schizophrenia symptomatology.

- Dichotomous outcomes: the effect size for dichotomous outcomes will be the risk ratio (RR) and its $95 \%$ CIs.

\section{Relative treatment ranking}

We will estimate the probability for each intervention to be ranked at each possible place, given the relative effect sizes as estimated in NMA. As described in Salanti et $a l,{ }^{24}$ we will obtain a hierarchy of the competing interventions using the surface under the cumulative ranking curve (SUCRA) and mean ranks. SUCRA values will be expressed as percentage, showing the relative probability of an intervention to be among the best options.

\section{Dealing with missing outcome data and missing statistics}

For continuous outcomes we will extract data for all randomised patients, if possible, and we will give preference to data based on mixed-effect models of repeated measurements of multiple imputations over last-observation-carried-forward data.

We will use published SDs, where available. When SEs instead of SDs are presented, the former will be converted to SDs. ${ }^{25}$ If both are missing, we will estimate SDs from $\mathrm{P}$ values or CIs, as described in Section 7.7.3 of the Cochrane Handbook for Systematic Reviews. ${ }^{26}$ If none of these options are viable, we will contact the original authors. When no information can be obtained we will derive SDs from those of the other studies using a validated imputation technique. ${ }^{25}$

For dichotomous outcomes, everyone allocated to the intervention will be counted whether they completed the follow-up or not. If the authors applied such a strategy, we will use their results. If the original authors presented only the results of the per-protocol or completer population, we will assume that those participants lost to follow-up would not have changed in a given outcome. In terms of efficacy, this means that they would be conservatively considered to have not responded to treatment or control. In terms of tolerability, it would mean that participants would not have developed a side-effect.

\section{Risk of bias assessment}

Risk of bias will be assessed for each included study using the Cochrane Collaboration 'risk of bias' tool. ${ }^{26}{ }^{27}$ The following domains will be considered:

1. Sequence generation: was the allocation sequence adequately generated?

2. Allocation concealment: was allocation adequately concealed?

3. Blinding of participants: was knowledge of the allocated treatment adequately prevented during the study? Given the peculiarity of the included studies, in which the therapist cannot be blind, we will consider under this item only if a way was found to keep patients unaware of the treatment they were receiving (even if we expect this will not be likely).

4. Blinding of outcome assessors: were outcomes evaluated by blind raters? Were adequate measures taken to prevent them from discovering treatment allocation during the study?

5. Incomplete outcome data: were incomplete outcome data adequately addressed?

6. Selective reporting: are reports of the study free from suggestion of selective outcome reporting?

7. Researcher's allegiance: do the researchers involved have a vested interest for the psychological treatment under investigation? We will additionally consider this point as possible source of bias, since it has been claimed to be relevant in trials on psychological interventions. ${ }^{28-30}$ An evaluation of high risk of bias will be given, for example, when the authors are founders of the therapy or have written a manual for that therapy.

A description of what was reported about the same domains in each study will be provided, and a judgement on the risk of bias will be made for each one of them, based on the following three categories: 'high risk of bias', 'low risk of bias' and 'unclear risk of bias' where information are not sufficient to make a judgement. Two independent review authors will assess the risk of bias in the selected studies. Any disagreement will be resolved through discussion. Where necessary, the authors of the studies will be contacted for further information. Studies will be classified as having low risk of bias if none of the domains above were rated as high risk of bias and three or less were rated as unclear risk; moderate if one was 
rated as high risk of bias or none was rated as high risk of bias, but four or more were rated as unclear risk and all other cases will be assumed to pertain to high risk of bias. ${ }^{31}$ We will not include studies in the data analyses whose sequence generation was at high risk of bias (eg, randomisation by the date of birth or day of the week). Effects of high risk of bias in the other domains will be analysed by sensitivity analyses.

\section{Data analysis}

\section{Characteristics of the included studies}

We will produce descriptive statistics and study population characteristics across all eligible trials, describing the types of comparisons and other clinical or methodological variables, such as age, duration of illness, comedication, country, duration of study and number of sessions.

\section{Two-step procedure}

In a first step, we will perform series of conventional pairwise meta-analyses by combining studies that compared the same interventions, including the comparison between active treatments and the different control arms. In subgroups with very few RCTs available or if the requirements of NMA are not met, it can be that NMA will not be appropriate and, in this case, conventional pairwise meta-analysis will be the most straightforward approach. As heterogeneity is likely, a random-effects model will be used. In a second step, we will then perform a NMA within a frequentist framework.

\section{Assessment of heterogeneity}

The heterogeneity (variability in relative treatment effects within the same treatment comparison) will be measured with the tau-squared (the variance of the random-effects distribution). The heterogeneity variance will be assumed common across the various treatment comparisons (grouped by comparison type) and the empirical distributions will be used to characterise the amount of heterogeneity as low, moderate or high using the first and third quantiles. ${ }^{32-34}$ Potential reasons for heterogeneity will be explored by subgroup analysis (see below).

\section{Assessment of the transitivity assumption}

Joint analysis of treatments can be misleading if the network is substantially intransitive. We assume that patients who fulfil the inclusion criteria outlined in criteria for considering studies for this review section are equally likely to be randomised to any of the interventions that we plan to compare. We will need to investigate the distribution of clinical and methodological variables that can act as effect modifiers across treatment comparisons. ${ }^{35}$ We have maximised the chances of transitivity in our network with regard to clinical variables by limiting our samples to participants with schizophrenia and excluding specific subgroups like first-episode patients or patients with prevalent negative symptoms. Other clinical or methodological variables that may influence the efficacy of psychological interventions include administration mode and frequency of the treatment (like number of sessions and experience of the therapist), baseline severity (see 'Investigation of heterogeneity and inconsistency') and blinding, which will also be assessed in sensitivity analyses. We will investigate if these variables are similarly distributed across studies grouped by comparison. The comparability of studies comparing the intervention with treatment as usual or waiting-list conditions with those that provide head-to-head evidence will be examined carefully.

Network meta-analysis

NMA combines direct and indirect evidence for all relative treatment effects and can therefore provide estimates with maximum power and increased precision. ${ }^{36}$ If the collected studies appear to be sufficiently similar with respect to the distribution of effect modifiers (refer the Assessment of transitivity assumption section), we will conduct a random-effects NMA to synthesise all evidence for each outcome, and obtain a comprehensive ranking of all treatments. We will assume a single heterogeneity parameter for each network. We will present the summary SMDs or RRs for all pairwise comparisons in a league table. We will also estimate the prediction intervals to assess how much the common heterogeneity affects the relative effect with respect to the extra uncertainty anticipated in a future study. To rank the various treatments for each outcome, we will use the SUCRA and the mean ranks.

\section{Assessment of inconsistency}

The strategical and conceptual evaluation of transitivity will be supplemented with a statistical evaluation of consistency, the agreement between direct and indirect evidence. We will employ local as well as global methods to evaluate consistency. ${ }^{37}$ Local methods detect 'hot spots' of inconsistency, evidence loops that are inconsistent or comparisons for which direct and indirect evidence disagree. We will employ a method that separates direct evidence from indirect evidence provided by the entire network and then evaluate the agreement of these two pieces of evidence. ${ }^{38}$ We will also evaluate consistency in the entire network by calculating the design-by-treatment interaction test and I-squared for network heterogeneity, inconsistency, and for both. ${ }^{39}$ Tests for inconsistency are known to have low power, and empirical evidence has suggested that $10 \%$ of evidence loops published in the medical literature are expected to be inconsistent. ${ }^{40}$ Therefore, interpretation of the statistical inference about inconsistency will be carried out with caution and possible sources of inconsistency will be explored even in the absence of evidence for inconsistency.

\section{Investigation of heterogeneity and inconsistency}

We expect small amounts of heterogeneity and inconsistency to be present given the variety of study settings we plan to include. The following potential effect modifiers of the primary outcome will be explored by subgroup analyses: 
A. Number of sessions,

B. Study duration,

C. Setting: individual versus group,

D. Expertise of the therapist,

E. Baseline severity (PANSS or BPRS score at baseline),

F. Different types of patients, with a different clinical outline concerning symptoms (if identified).

\section{Sensitivity analyses}

We will explore the following sensitivity analyses by excluding:

A. Studies in which the outcome assessor was not blind (open studies);

B. Studies that presented only completer analyses;

C. Studies characterised as pertaining to high risk of bias;

D. Studies with high risk of bias in researchers' allegiance;

E. Studies focused on treatment resistant patients (study defined);

F. Studies with a non-active comparison group.

\section{Publication bias}

We will first examine funnel plots of pairwise NMAs if there are 10 or more studies included. We will also explore the association between study size and effect size with a comparison-adjusted funnel plot that has been adapted to NMA. ${ }^{41}$

\section{Evaluating the quality of the evidence}

The quality of evidence contributing to each network estimate will be evaluated using an adaptation of the Grading of Recommendations Assessment, Development and Evaluation framework specifically developed for NMA. ${ }^{37}$ We will characterise the credibility of a body of evidence based on the study limitations, imprecision, heterogeneity/inconsistency, indirectness and publication bias.

\section{Statistical software}

The analysis and presentation of results will be performed using the Stata packages network and network_graphs, and the $\mathrm{R}$ package netmeta.

Acknowledgements The authors acknowledge the collaborators Samantha Roberts in helping us to conduct the literature searches, and Maximilian Huhn, Johannes Schneider-Thoma, Marc Krause and Costanza Carmi for their help and suggestions.

Collaborators Samantha Roberts; Maximilian Huhn, Johannes Schneider-Thoma; Marc Krause; Costanza Carmi.

Contributors IB and SL designed this study, drafted and critically revised the protocol. IB will screen search results for inclusion, conduct data extraction and data analysis and draft the final manuscript. SL will assist with data extraction and analysis and revise the final manuscript. CR and SW will screen search results for inclusion and conduct data extraction. GS provided substantial methodological advice in planning the study and will assist with data analysis. CB and TAF contributed with clinical and methodological input in planning the study. All authors contributed to and have approved the final manuscript.

Funding This project has received funding from the European Union's Horizon 2020 Research and Innovation Programme under the Marie Skłodowska-Curie grant agreement no 701717. This work was also supported by the German Research Foundation (DFG) and the Technical University of Munich within the Open Access Publishing Funding Programme.

Disclaimer The funder had no role in developing the protocol.
Competing interests $\mathrm{SL}$ in the past 3 years has received honoraria for consulting from Roche, TEVA, Otsuka, Lundbeck and LB Pharma; for lectures from Otsuka, Lundbeck, Janssen, ICON, Lilly, Sanofi Aventis, AOP Orphan, Roche and Servier; and for a publication from Roche. TAF has received lecture fees from Eli Lilly, Janssen, Meiji, Mitsubishi-Tanabe, MSD and Pfizer and consultancy fees from Takeda Science Foundation. He has received royalties from Igaku-Shoin and Nihon Bunka Kagaku-sha publishers. He has received research support from Mochida and Mitsubishi-Tanabe.

Patient consent Not required.

Provenance and peer review Not commissioned; externally peer reviewed.

Open Access This is an Open Access article distributed in accordance with the Creative Commons Attribution Non Commercial (CC BY-NC 4.0) license, which permits others to distribute, remix, adapt, build upon this work non-commercially, and license their derivative works on different terms, provided the original work is properly cited and the use is non-commercial. See: http://creativecommons.org/ licenses/by-nc/4.0/

(C) Article author(s) (or their employer(s) unless otherwise stated in the text of the article) 2018. All rights reserved. No commercial use is permitted unless otherwise expressly granted.

\section{REFERENCES}

1. Vos T, Flaxman AD, Naghavi M, et al. Years lived with disability (YLDs) for 1160 sequelae of 289 diseases and injuries 1990-2010: a systematic analysis for the Global Burden of Disease Study 2010. Lancet 2012;380:2163-96.

2. Leucht S, Cipriani A, Spineli L, et al. Comparative efficacy and tolerability of 15 antipsychotic drugs in schizophrenia: a multipletreatments meta-analysis. Lancet 2013;382:951-62.

3. National Collaborating Centre for Mental Health. Core interventions in the treatment and management of schizophrenia in adults in primary and secondary care (Clinical Guideline CG82). London 2009.

4. Buchanan RW, Kreyenbuhl J, Kelly DL, et al. The 2009 schizophrenia PORT psychopharmacological treatment recommendations and summary statements. Schizophr Bull 2010;36:71-93.

5. Pinquart M, Oslejsek B, Teubert D. Efficacy of systemic therapy on adults with mental disorders: A meta-analysis. Psychother Res 2016;26:241-57.

6. Ruddy R, Milnes D. Art therapy for schizophrenia or schizophrenialike illnesses. Cochrane Database Syst Rev 2005;4:CD003728.

7. Jones C, Hacker D, Cormac I, et al. Cognitive behaviour therapy versus other psychosocial treatments for schizophrenia. Cochrane Database Syst Rev 2012;4:CD008712.

8. Lutgens D, Gariepy G, Malla A. Psychological and psychosocial interventions for negative symptoms in psychosis: systematic review and meta-analysis. Br J Psychiatry 2017;210:324-32.

9. Zimmermann G, Favrod J, Trieu VH, et al. The effect of cognitive behavioral treatment on the positive symptoms of schizophrenia spectrum disorders: a meta-analysis. Schizophr Res 2005;77:1-9.

10. Turner DT, van der Gaag M, Karyotaki E, et al. Psychological interventions for psychosis: a meta-analysis of comparative outcome studies. Am J Psychiatry 2014;171:523-38.

11. Hutton B, Salanti G, Caldwell DM, et al. The PRISMA extension statement for reporting of systematic reviews incorporating network meta-analyses of health care interventions: checklist and explanations. Ann Intern Med 2015;162:777-84.

12. Shamseer $L$, Moher $D$, Clarke $M$, et al. Preferred reporting items for systematic review and meta-analysis protocols (PRISMA-P) 2015: elaboration and explanation. BMJ 2015;349:97647.

13. Egger M, Zellweger-Zähner T, Schneider M, et al. Language bias in randomised controlled trials published in English and German. Lancet 1997;350:326-9.

14. In: Wu T, Li Y, Liu G, Bian Z, Li J, et al. eds. Investigation of authenticity of 'claimed' randomized controlled trials (RCTs) and quality assessment of RCT reports published in China, 2006.

15. Woodhead M. $80 \%$ of China's clinical trial data are fraudulent, investigation finds. BMJ 2016;355:i5396.

16. Carpenter WT, Buchanan RW. Schizophrenia. N Engl J Med 1994;330:681-90.

17. Adams CE, Coutinho E, Davis JM, et al; The cochrane library. Chichester, UK: John Wiley \& Sons Ltd, 2011.

18. Leucht S, Leucht C, Huhn M, et al. Sixty years of placebo-controlled antipsychotic drug trials in acute schizophrenia: systematic review, bayesian meta-analysis, and meta-regression of efficacy predictors. Am J Psychiatry 2017;174:927-42. 
19. Zhu Y, Li C, Huhn M, et al. How well do patients with a first episode of schizophrenia respond to antipsychotics: a systematic review and meta-analysis. Eur Neuropsychopharmacol 2017;27:835-44.

20. Furukawa TA, Noma $\mathrm{H}$, Caldwell DM, et al. Waiting list may be a nocebo condition in psychotherapy trials: a contribution from network meta-analysis. Acta Psychiatr Scand 2014;130:181-92.

21. Marshall M, Lockwood A, Bradley C, et al. Unpublished rating scales: a major source of bias in randomised controlled trials of treatments for schizophrenia. Br J Psychiatry 2000;176:249-52.

22. Vaughan B, Goldstein MH, Alikakos M, et al. Frequency of reporting of adverse events in randomized controlled trials of psychotherapy vs. psychopharmacotherapy. Compr Psychiatry 2014;55:849-55

23. Linden M, Schermuly-Haupt ML, Definition S-HM-L. Definition, assessment and rate of psychotherapy side effects. World Psychiatry 2014;13:306-9.

24. Salanti G, Ades AE, loannidis JP. Graphical methods and numerical summaries for presenting results from multiple-treatment metaanalysis: an overview and tutorial. J Clin Epidemiol 2011;64:163-71.

25. Furukawa TA, Barbui C, Cipriani A, et al. Imputing missing standard deviations in meta-analyses can provide accurate results. J Clin Epidemiol 2006;59:7-10

26. Higgins JPT. Cochrane handbook for systematic reviews of interventions. Version 5.1.0, 2011. (accessed Mar 2011).

27. Higgins JPT, Altman DG, Sterne JAC. et alAssessing risk of bias in included studies. In: Higgins JPT, Churchill R, Chandler J, Cumpston MS, . Cochrane handbook for systematic reviews of interventions version 5.2.0, 2017. (accessed Jun 2017).

28. Dragioti E, Dimoliatis I, Evangelou E. Disclosure of researcher allegiance in meta-analyses and randomised controlled trials of psychotherapy: a systematic appraisal. BMJ Open 2015;5:e007206.

29. Lieb K, von der Osten-Sacken J, Stoffers-Winterling J, et al. Conflicts of interest and spin in reviews of psychological therapies: a systematic review. BMJ Open 2016;6:e010606.

30. Munder T, Brütsch $\mathrm{O}$, Leonhart $\mathrm{R}$, et al. Researcher allegiance in psychotherapy outcome research: an overview of reviews. Clin Psychol Rev 2013;33:501-11.

31. Furukawa TA, Salanti G, Atkinson LZ, et al. Comparative efficacy and acceptability of first-generation and second-generation antidepressants in the acute treatment of major depression: protocol for a network meta-analysis. BMJ Open 2016;6:e010919.

32. Turner RM, Davey J, Clarke MJ, et al. Predicting the extent of heterogeneity in meta-analysis, using empirical data from the cochrane database of systematic reviews. Int J Epidemiol 2012:41:818-27.

33. Rhodes KM, Turner RM, Higgins JP. Empirical evidence about inconsistency among studies in a pair-wise meta-analysis. Res Synth Methods 2016;7:346-70.

34. Rhodes KM, Turner RM, White IR, et al. Implementing informative priors for heterogeneity in meta-analysis using meta-regression and pseudo data. Stat Med 2016;35:5495-511.

35. Salanti G. Indirect and mixed-treatment comparison, network, or multiple-treatments meta-analysis: many names, many benefits, many concerns for the next generation evidence synthesis tool. Res Synth Methods 2012;3:80-97.

36. Salanti G, Higgins JP, Ades AE, et al. Evaluation of networks of randomized trials. Stat Methods Med Res 2008;17:279-301.

37. Salanti G, Del Giovane C, Chaimani A, et al. Evaluating the quality of evidence from a network meta-analysis. PLoS One 2014;9:e99682.

38. Dias S, Welton NJ, Caldwell DM, et al. Checking consistency in mixed treatment comparison meta-analysis. Stat Med 2010;29:932-44.

39. Higgins JP, Jackson D, Barrett JK, et al. Consistency and inconsistency in network meta-analysis: concepts and models for multi-arm studies. Res Synth Methods 2012;3:98-110.

40. Veroniki AA, Vasiliadis HS, Higgins JP, et al. Evaluation of inconsistency in networks of interventions. Int J Epidemiol 2013;42:332-45.
41. Chaimani A, Salanti G. Using network meta-analysis to evaluate the existence of small-study effects in a network of interventions. Res Synth Methods 2012;3:161-76.

42. Naeem F, Asmer MS, Khoury B, et al. Acceptance and commitment therapy for schizophrenia and related disorders. Cochrane Database Syst Rev 2015;313.

43. Gray R, Bressington D, Ivanecka A, et al. Is adherence therapy an effective adjunct treatment for patients with schizophrenia spectrum disorders? A systematic review and meta-analysis. BMC Psychiatry 2016;16:90.

44. Marshall M, Lockwood A. Assertive community treatment for people with severe mental disorders. Cochrane Database Syst Rev 2000;(2):CD001089.

45. Dieterich $\mathrm{M}$, Irving $\mathrm{CB}$, Bergman $\mathrm{H}$, et al. Intensive case management for severe mental illness. Cochrane Database Syst Rev 2017;(1):CD007906.

46. Jauhar S, McKenna PJ, Radua J, et al. Cognitive-behavioural therapy for the symptoms of schizophrenia: systematic review and meta-analysis with examination of potential bias. Br J Psychiatry 2014;204:20-9.

47. van der Gaag M, Valmaggia LR, Smit F. The effects of individually tailored formulation-based cognitive behavioural therapy in auditory hallucinations and delusions: a meta-analysis. Schizophr Res 2014; 156:30-7.

48. Hazell CM, Hayward M, Cavanagh $\mathrm{K}$, et al. A systematic review and meta-analysis of low intensity CBT for psychosis. Clin Psychol Rev 2016;45:183-92

49. Kennedy L, Xyrichis A. Cognitive behavioral therapy compared with non-specialized therapy for alleviating the effect of auditory hallucinations in people with reoccurring schizophrenia: a systematic review and meta-analysis. Community Ment Health $\mathrm{J}$ 2017:53:127-33

50. Cella M, Preti A, Edwards $C$, et al. Cognitive remediation for negative symptoms of schizophrenia: A network meta-analysis. Clin Psychol Rev 2017;52:43-51.

51. Ren J, Xia J. Dance therapy for schizophrenia. Cochrane Database Syst Rev 2013;10:CD006868.

52. Pitschel-Walz G, Leucht S, Bäuml J, et al. The effect of family interventions on relapse and rehospitalization in schizophrenia--a meta-analysis. Schizophr Bull 2001;27:73-92.

53. Pharoah F, Mari J, Rathbone J, et al. Family intervention for schizophrenia. Cochrane Database Syst Rev 2010;12:CD000088.

54. Orfanos S, Banks C, Priebe S. Are group psychotherapeutic treatments effective for patients with schizophrenia? A systematic review and meta-analysis. Psychother Psychosom 2015;84:241-9.

55. Roder V, Mueller DR, Mueser KT, et al. Integrated psychological therapy (IPT) for schizophrenia: is it effective? Schizophr Bull 2006;32(Suppl 1):S81-93.

56. Eichner C, Berna F. Acceptance and Efficacy of Metacognitive Training (MCT) on positive symptoms and delusions in patients with schizophrenia: a meta-analysis taking into account important moderators. Schizophr Bull 2016;42:952-62.

57. Aust J, Bradshaw T. Mindfulness interventions for psychosis: a systematic review of the literature. J Psychiatr Ment Health Nurs 2017;24:69-83

58. Geretsegger M, Mössler KA, Bieleninik $七$, et al. Music therapy for people with schizophrenia and schizophrenia-like disorders. Cochrane Database Syst Rev 2017;5:CD004025.

59. Malmberg L, Fenton M. Individual psychodynamic psychotherapy and psychoanalysis for schizophrenia and severe mental illness. Cochrane Database Syst Rev 2001(3):CD001360.

60. Pekkala E, Merinder L. Psychoeducation for schizophrenia. Cochrane Database Syst Rev 2000;(4):CD002831.

61. Almerie MQ, Okba Al Marhi M, Jawoosh M, et al. Social skills programmes for schizophrenia. Cochrane Database Syst Rev 2015;6:CD009006.

62. Buckley LA, Maayan N, Soares-Weiser K, et al. Supportive therapy for schizophrenia. Cochrane Database Syst Rev 2015;4:CD004716. 